\title{
Peripheral Lymph Node Addressin
}

National Cancer Institute

\section{Source}

National Cancer Institute. Peripheral Lymph Node Addressin. NCI Thesaurus. Code C106319.

A group of sulfated sialomucins that share a carbohydrate epitope synthesized by Nacetylglucosamine 6-O-sulfotransferase. The glycoproteins with this modification include sulfated ligands for L-selectin and are involved in tethering and rolling of lymphocytes along high endothelial venules (HEV) of lymphoid tissues. 